\title{
A Study of English Writing Instruction with Use of Multimedia
}

\author{
Rong-Wang Hsu, Chia-Ying Chang, and Hann-Jang Ho
}

\begin{abstract}
Educational technology is nowadays prevailing worldwide. By using and managing appropriate online resources, students are able to facilitate learning and improve performance. With Internet technology and multimedia, the style of teaching and learning is gradually changing. In the traditional classroom, teaching materials are amplified by using technology and multimedia, such as videos, images and sound. The development in multimedia brought the transitions in the forms of teaching materials and instructional tools. The purpose of this research is to investigate effect of teaching English writing with multimedia to 3rd graders in senior high school in Taiwan. With descriptive-qualitative design, this paper aims to answer how multimedia effect students' learning results by looking into the topical structures employed in students' descriptive writings after a series of instruction implemented with multimedia in English writing classes. Based on the above conclusions, it is highly recommended that writing instruction combined with multimedia be introduced as one of writing approaches in Taiwan in order to enhance the coherence in EFL writing.
\end{abstract}

Index Terms-English writing, multimedia, English instruction, educational technology.

\section{INTRODUCTION}

\section{A. Background}

Recently, the increasing interest in writing instruction has highlighted the need for exploring the elements of constructing a successful writing. According to Almaden [1], a good writing requires logically consistent ideas in order for the writer to compose a coherent piece of discourse that is comprehensible to the intended audience may be. However, overly emphasis has been put on creating the text at the level of local choice of grammar and vocabulary while an overall discourse structure has been neglected. In attempts to explore the coherence problems in student's ESL/EFL writings, Lautamatti [2] proposed a textlinguistic research method on the basis of the discourse organization patterns that investigates how sentence topics combine and progress contributing to the coherence of a text. This study is expected to utilize Lautamatti's topical structure analysis as an instruction tool to help Taiwanese senior-high students enhance the coherence in English compositions.

\section{B. Motivation}

For decades, instructors and students in Taiwan believe that a well-written composition lies in grammatically

Manuscript received October 13, 2019; revised January 20, 2020.

The authors are with Department of Applied Digital Media, Wufeng University, Taiwan (e-mail: ronger@wfu.edu.tw, andreanchang@gmail.com, hhj@wfu.edu.tw). accuracy. Likewise, textbooks put major emphasis on teaching sentence-level grammar rather than coherence in a broader sense [3]. Writing lessons overly emphasize the drills of sentences patterns, substitutions, completion or imitation of sentences and so on [4]. Teachers are suggested to focus mainly on separated sentences rather than the whole idea of the writing. Accordingly, Lautamatti explores the coherence of whole discourse in terms of the relations between discourse topic and subsequent topics. Lautamatti refers coherence to the organization of information in discourse and the development of the semantic content. In order to investigate coherence in text, she proposed Topical Structure Analysis (TSA), which is one method writers have adopted to achieve coherence in English writing. According to Almaden [1], "The use of TSA as device in investigating the progressions in writing has been validated by several researchers as a clear demonstration of style and strategy in linking ideas within paragraphs and reflection of thought progression". Therefore, the purpose of the study is trying to develop an effective tool for writers to connect and organize ideas. By utilizing Lautamatti's topical structure analysis as an instruction, students can develop with higher awareness of the coherence in writing. Therefore, students can write English compositions with coherence.

\section{Purpose}

In attempts to explore the coherence problems in student's ESL/EFL writings, Lautamatti proposed a textlinguistic research method on the basis of the discourse organization patterns that investigates how sentence topics combine and progress contributing to the coherence of a text. This study is expected to utilize Lautamatti's topical structure analysis as an instruction tool to help Taiwanese senior-high students enhance the coherence in English compositions.

\section{LITERATURE REVIEW}

\section{A. Coherence in English Writing}

Coherence refers to the way a text makes sense to the reader through the relevance and accessibility of its configuration of concepts and ideas [5]. Various approaches of coherence structure have been discussed in many studies [6]. To conclude the scholar [7], [8] definition of coherence, coherence lies beyond the surface structure of a text and it is often characterized as a text with a discourse theme accompanied by a set of related ideas connected logically by cohesive devices to convey a clear information structure. The main concern of coherence is about how given-new information is distributed within and without sentences rather than the surface grammatical structure. Scholar [9], [10] 
refers to the way in which clauses are structured to present the writer's assumptions about what is known (given) or new to the reader. That is, given information refers to information that has been introduced beforehand while new information refers to information that is introduced for the first time. To achieve coherence, given information should be put first and new information comes last. Thus, given-new principle shows how the information is organized coherently to enhance communication between the writer and the reader. In order to write a well-composed writing, the coherence matters in writing instruction.

\section{B. Topical Structure Analysis (TSA)}

Accordingly, Lautamatti explores the coherence of whole discourse in terms of the relations between discourse topic and subsequent topics. Lautamatti refers coherence to the organization of information in discourse and the development of the semantic content. In order to investigate coherence in text, she proposed Topical Structure Analysis (TSA), which is one method writers have adopted to achieve coherence in English writing. TSA has been acknowledged by researchers in determining thematic development in writings. Some researchers [11], [12] also indicated that investigation of topic in discourse is a more promising direction for ESL/EFL studies on coherence issue [13], [14]. Likewise, scholar [8], [15] considered Lautimatti's work as an important tool applicable to the analysis of students writing for three reasons. One is that the framework provides a functionally based taxonomy of topical and non-topical linguistic material in a sentence and the categories help to explain the function of each segment in sentence. The other is that the specification of topical information is not merely given-new information. Another reason is that the analysis shows that the topical progression may be a more readable than others. Therefore, Topical Structure Analysis (TSA) has been acknowledged by several researchers as a more satisfying tool for evaluating coherence.

\section{TSA Employed in Teaching}

Studies have acknowledged TSA as a promising criterion of coherence and TSA has direct relation to the quality of writings [16]. Many studies employed TSA as a tool of examining the coherence of writing [14], [16]. Additionally, TSA is used as a revision tool in students' writing. Scholar [17] employed TSA as a revision strategy for ESL writers and positively improved writing coherence by utilizing TSA. Similarly, Scholar [18] in her case study helps student develop coherence based on TSA. Scholar [19], meanwhile, acknowledged TSA as an effective way in improving writing coherence. In conclusion, TSA is not only an effective tool for teachers to investigate the coherence problems but an effective revision strategy for students to improve writing ability. Consequently, a mended writing instruction concerning to students' problem can be provided. Moreover, TSA can also be taught as a reference for students to examine their writing quality as well as to improve their writing.

\section{Related Studies of TSA in Teaching}

Studies have acknowledged TSA as a promising criterion of coherence and TSA has direct relation to the quality of writings [16]. Many studies employed TSA as a tool of examining the coherence of writing [14], [16]. Scholar [20] in their studies concluded that essays in which topical structuring is properly accomplished are more cohesive and coherent. Likewise, Park \& Nam [21] imply that an explicit instruction of theme structure should be provided in later units of curriculum. That is, thematic structure is helpful for students to produce logically sentences. Consequently, the students' familiarity with the TSA would contribute to enhancing coherence in their written essays. Additionally, TSA is used as a revision tool in students' writing. Connor and Farmer [17] employed TSA as a revision strategy for ESL writers and positively improved writing coherence by utilizing TSA. Similarly, Chiu [18] in her case study helps student develop coherence based on TSA. Scholar [19], meanwhile, acknowledged TSA as an effective way in improving writing coherence. Also, Shabana [22] suggests that it is recommended that instructors use the TSA as a strategy to assess and teach both intermediate and advanced academic writing courses. With TSA as an assessment, Scholar [19], [21] indicates the overload of topic sequencing may lead to disorientation of the focus of the writing. In this way, TAS can be utilized as a useful assessment for writers to examine the coherence of the writing. In conclusion, TSA is not only an effective tool for teachers to investigate the coherence problems but an effective revision strategy for students to improve writing ability. Consequently, a mended writing instruction concerning to students' problem can be provided. Moreover, TSA can also be taught as a reference for students to examine their writing quality as well as to improve their writing.

\section{METHODOLOGY}

\section{A. The Research Questions}

1) The effect of teaching English writing with multimedia to $3^{\text {rd }}$ graders in senior high school in Taiwan.

2) The frequency of topical progressions between control group essays and experimental group essays.

3) The analysis of topical progressions carried out in the compositions.

\section{B. The Teaching Procedures and Materials}

The teaching procedure is divided into two sessions. One is presented by slides, the other is presented through online tutoring videos [23]. Both teaching materials are designed based on the protocol of the theory: Lautamatti's Topical Structure Analysis [24]. In the first session, the material is presented through slides with oral instruction of the teacher. The following images are extracted from the power point. Slides 1 is to introduce the structure of writing. This part is aimed to help the writers to construct a well-formed structure. The second part is to introduce the destruction web. This part uses a diagram to help writers brainstorm. The design of this diagram meets the idea of Lautamatti's Topical Structure. By using this diagram, the related ideas are connected to the main topic. After the brainstorming, the writer writes the first draft, the teacher revised the writing with the theory based on Lautamatti's Topical Structure Analysis. This session is aimed to help writers understand how sentence topics and 
discourse topic combine and progress contributing to the coherence of a text. The material contains two samples to help writers fully understand how to apply Lautamatti's Topical Structure Analysis in their writings.

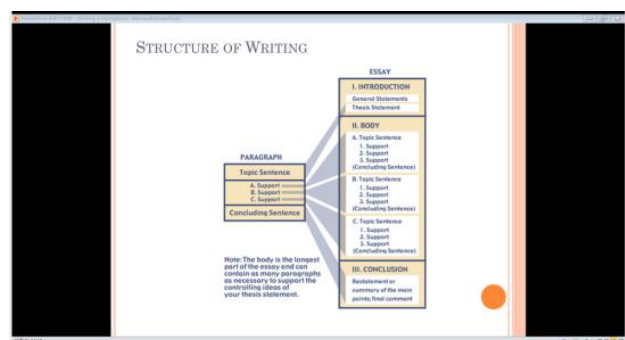

Slide1.1. Structure of writing.

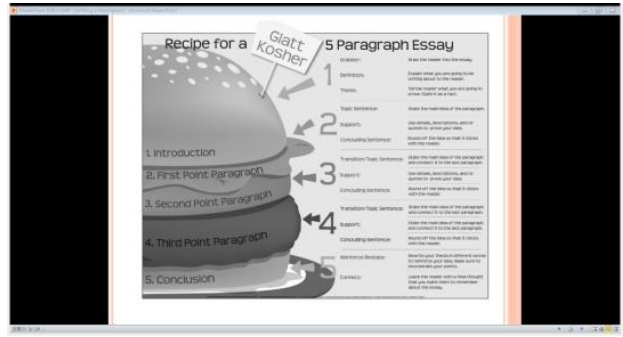

Slide 1.2. Structure of writing.

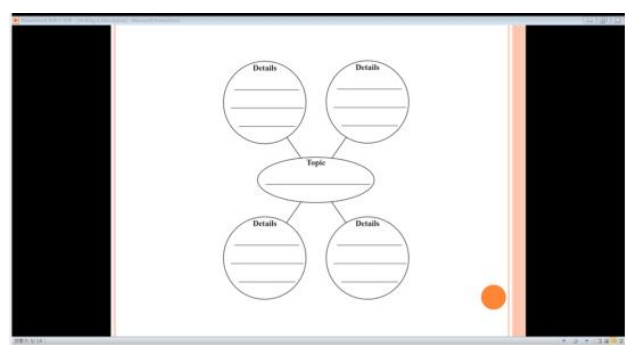

Slide 2. Description web.

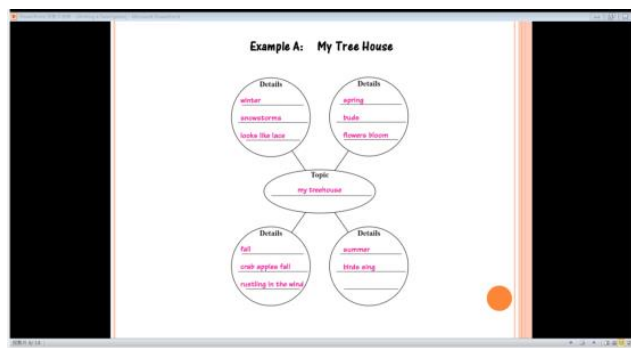

Slide 3. Example of description web.

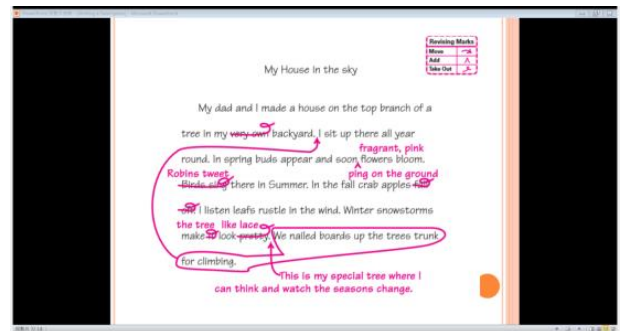

Slide 4. First draft by using TSA.

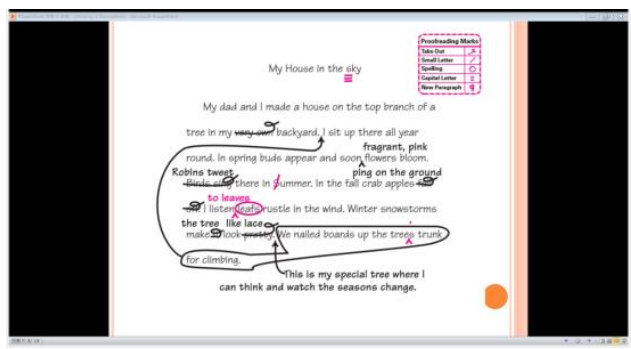

Slides 5. Final draft.

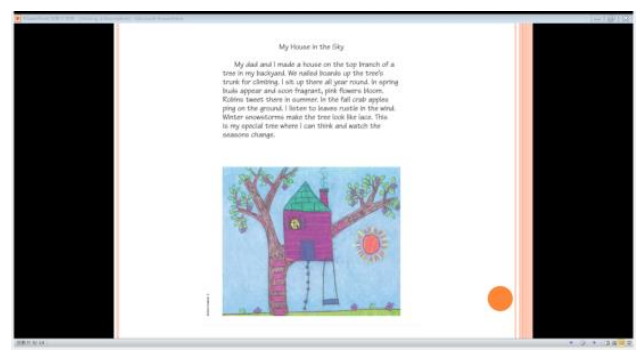

Slide 5. Samples of writing.

The other session is presented by a video, which is also designed based on the theory of idea development. The video is explained with the tutor's lecture in the video. This video aims to enhance the impression of Lautamatti's Topical Structure Analysis. This part uses structure of writing and the brainstorming skill to help students construct a writing. With the lecture in the video, students are able to know how to make their ideas organized. The following are images extracted from the video.

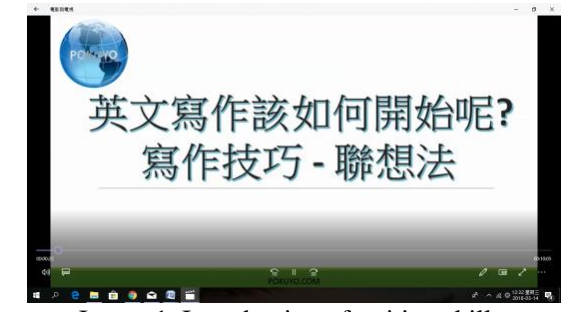

Image 1. Introduction of writing skills.

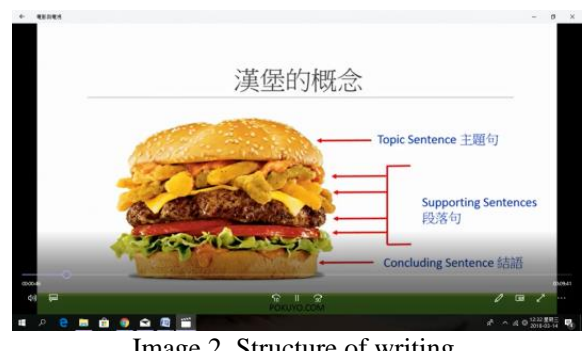

Image 2. Structure of writing.

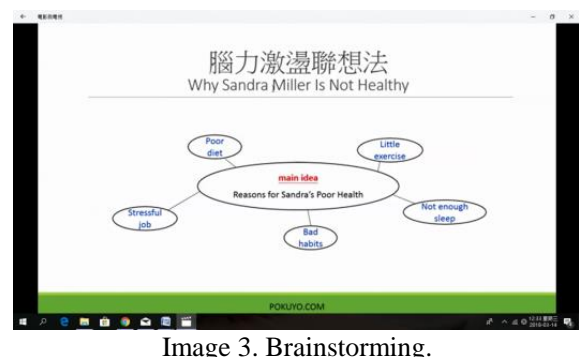

\section{RESEARCH IMPLEMENTATION}

\section{A. Background of Testers}

The study was conducted from two classes from a senior high school in Taiyuan in March in 2018. The English proficiency of the subjects was general. The subjects were a total 60 third- year students, who have learned English for at least five years. In the first grade, writing lessons focus on grammar and sentence combination practices. In the second grade, they have undergone series of Chinese-English translation training. In the third grade, they were asked to write paragraphs. General instruction of English writing structure was taught during the third grade. For example, they 
have been taught that a formal English writing should be composed of introduction-body-conclusion parts. Generally speaking, students write 10 pieces of writing in a semester. Writing material used here is "Fun writing through pictures". The original instruction of writing focuses majorly on the content development with minor emphasis on topical progression. Before giving a writing task, the teacher would provide paper handout explaining the basic writing structure which allow students to follow. The relation between sentences and the topic progressions of topics are seldom taught in class. Moreover, few examples are provided concerning the large number of paper handouts would cause a waste of paper resources.

\section{B. Implement of the Course}

The researcher teaches 2 sessions with TSA as an instruction to control group, which is consist of 30 students, to investigate the differences in terms of thematic progressions between the essays of control group and the essays of experimental group. These sessions are carried out in classroom equipped with computers. All the materials are presented through the screen in the front. On the other hand, the experimental group, which is also, consists of 30 students, undergoes traditional lecturing sessions without any assistant of multimedia equipment. The procedure of the sessions is shown as follows.

\section{Writing Task}

Undergoing the courses, students are asked to write an approximately 120 words descriptive composition in 50-minute class. During the writing session, the students are provided no interruption or guidance other than explanation of the prompt. The title of the writing is "My Best Friend." The purpose of choosing this genre is because students are in the early stage of writing and it is easier to start writing by expressing ideas. The title is chosen for it was believed that writings would be easier if beginner writers are given a topic most related to their life experience. Therefore, students are expected to express as much as possible without constraints.

\section{Control group}

$1^{\text {st }}$ session:

Slides introducing practices of Topical Progression

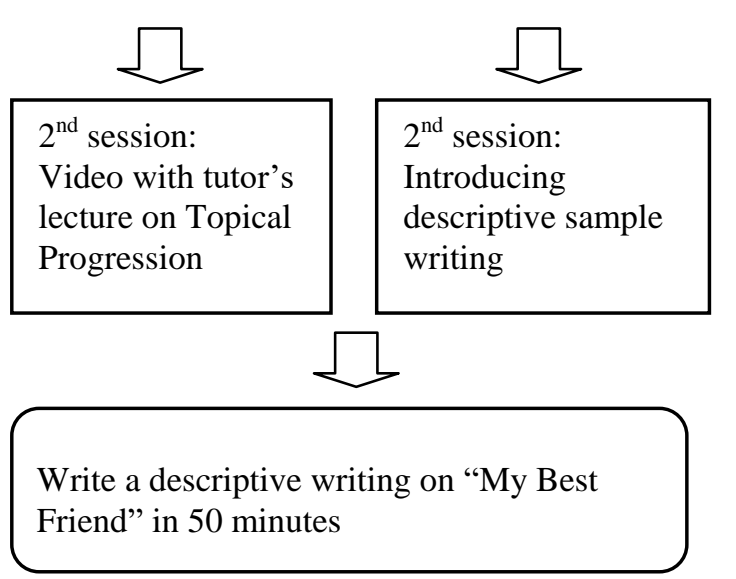

\section{Data Collection}

In order to investigate the effect of writing instruction with multimedia, the writings are collected and then examined by the resercher. The thematic progression is analyzed according to Topical Structure Analysis proposed by Lautamatti. I n order to examine the shift of topics and topic progressions and to investigate the text coherence. The following is the procedure of data collection.

\section{CONCLUSION}

In light of the finding of TSA implementation along with multimedia in writing courses, students are able to determine the focus of their writing more clearly and develop their subtopics more logically. Multimedia materials designed based on the protocol of Lautamatti's Topical Structure Analysis, as a result, is an effective tool for writers to connect and organize ideas. It is suggested that multimedia and online resources implemented as an instruction tool in Taiwanese high school. Lautamatti's Topical Structure Analysis can be introduced through multimedia in an easy way. Through technology and multimedia, difficult teaching materials are able to be comprehended and applied by beginning learners. Moreover, by utilizing Lautamatti's Topical Structure Analysis as an instruction, students can develop with higher awareness of the coherence in writing. Therefore, students can write English compositions with coherence by examining their first draft since it is a promising indicator of incoherence and digression. Lautamatti's Topical Structure Analysis (TSA) is not only a revision tool for students to edit their first draft, but a helpful indicator for instructors to assess incoherence in writing. In a word, with the use of multimedia, teachers can explain the abstract and difficult ideas with evident exemplification on the screen or computers. Therefore, multimedia is highly recommended as a teaching tool as well as a countable assessor in writing courses.

\section{CONFLICT OF INTEREST}

The authors declare no conflict of interest.

\section{AUTHOR CONTRIBUTIONS}

All authors had approved the final version.

\section{REFERENCES}

[1] D. Almaden, "An analysis of the topical structure of paragraphs written by Filipino students," The Asia-Pacific Education Research, vol. 15, no. 1, pp. 127-153, 2006.

[2] L. Lautamatti, "Observations on the development of the topic of simplified discourse," Writing across Languages, Analysis of L2 Texts, Reading, MA: Addison Wesley, 1987.

[3] I. Lee, "Teaching coherence to ESL students: A classroom inquiry," Journal of Second Language Writing, vol. 11, pp. 125-159, 2002.

[4] T. Silva, "L1 vs. L2 writing: ESL graduate students' perceptions," TESL Canada Journal, vol. 10, no. 1, pp. 27-48, 1992.

[5] K. Hyland, Metadiscourse, London, Continuum, 2005.

[6] A. M. Johns, "Coherence and academic writing: Some definitions and suggestions for teaching," Tesol Quarterly, vol. 20, no. 2, pp. 247-265, 1986.

[7] R. Kaplan, "Cultural thought patterns in intercultural education," Language Learning, vol. 16, pp. 1-20, 1966.

[8] S. O. Lin, T. S. Huang, C. I. Lin and K. T. Chen, "Construction and grading of English composition tests in the JCEE," in Proc. the Tenth 
Conference on English Teaching and Learning in the Republic of China, Taipei: Crane, pp. 557-609, 1993.

[9] A. M. Johns, "Coherence and academic writing: Some definitions and suggestions for teaching," TESOL Quarterly, vol. 20, no. 2, pp. 247-265, 1986.

[10] J. Firbas, "On the dynamics of written communication in the light of the theory of functional sentence perspective," Studying Writing. Linguistic Approaches, pp. 40-71, 1986.

[11] J. M. Simpson, "Topical structural analysis of academic paragraph in English and Spanish," Journal of Second Language Writing, vol. 9, no. 3, pp. 293-309, 2000

[12] W. Xing and Spencer, "Raising students' awareness of cross-cultural contrastive rhetoric in English writing via an e-learning course," Language Learning \& Technology, vol. 12, no. 2, pp. 71-93, 2008.

[13] E. Regala-Flores and K. Yin, "Topical structure analysis as an assessment tool in student academic writing," $3 L$ : Language, Linguistics, Literature ${ }^{\circledR}$, vol. 21, no. 1, 2015.

[14] M. Schneider and U. Connor, "Analyzing topical structure in ESL essays: Not all topics are equal," Studies in Second Language Acquisition, vol. 12, pp. 411-427, 1991.

[15] A. Raimes, "Out of the woods: Emerging traditions in the teaching of writing," TESOL Quarterly, vol. 25, no. 3, pp. 407-430, 1991.

[16] S. P. Witte, "Topical structure and writing quality: A study of the argumentative texts of college writers," Montreal, Canada, 1983

[17] U. Connor and M. Farmer, "Topical structure analysis as a revision strategy for ESL writers. In Barbara Kroll (Ed.)," Second Language Writing: Research Insights for the Classroom, Cambridge: CUP, 1990.

[18] Y. H. Chiu, "Coaching a student to develop coherence based on topical structure analysis: A case study," Journal of Language and Learning, vol. 2, pp. 154-170, 2004.

[19] J. J. Liu, "Intercultural rhetorical pattern differences in English argumentative writing," Journal of English as International Language, vol. 4, pp. 93-109, 2009.

[20] M. Kilic, B. Genç, and E. Badac, "Topical structure in argumentative essays of EFL learners and implications for writing classes," Journal of Language and Linguistic Studies, vol. 12, no. 2, pp. 107-116, 2016.

[21] K. Park and D. Nam, Analysis of Thematic Structure in L2 Writing a Systemic Functional Perspective, 2015

[22] N. O. Shabana, "Topical structure analysis assessing first-year Egyptian university students' internal coherence of their EFL writing," Assessing EFL Writing in the 21st Century Arab World, Palgrave Macmillan, Cham, 2018.
[23] R. Spack, "The rhetoric construction of multilingual students," TESOL Quarterly, vol. 31, pp. 765-774, 1997.

[24] A. M. Johns, "Coherence and academic writing: Some definitions and suggestions for teaching," Tesol Quarterly, vol. 20, no. 2, pp. 247-265, 1986.

Copyright $\odot 2020$ by the authors. This is an open access article distributed under the Creative Commons Attribution License which permits unrestricted use, distribution, and reproduction in any medium, provided the original work is properly cited (CC BY 4.0).

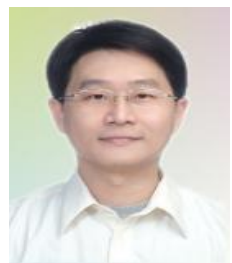

Rong-Wang Hsu was born in Kaohsiung, Taiwan. In 2007 , he studied doctorate in information technology at CYUT (Chaoyang University of Technology) of Science and Technology in Taiwan. He graduated in 2013 with a Ph.D. in information management. His main research fields are multimedia applications and information technology. His research interests include multimedia applications, marketing management Rong-Wang Hsu taught in the Department of Information Management of Wufeng University in Chiayi, Taiwan since 1999. He taught in the Department of Applied Digital Media in 2013. His teaching subjects focus on multimedia applications, and he is currently an assistant professor.

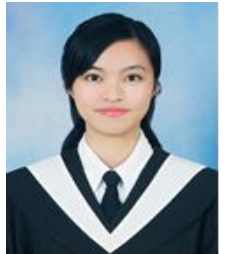

Chia-Ying Chang received her bachelor of English literature from Soochow University in 2008 and the master of applied digital media from WuFeng University in 2019. She is currently an English teacher of junior high school in Chiayi, Taiwan. Her interest is in the application of multimedia, especially in combination with English teaching.

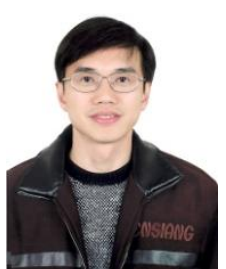

Hann-Jang Ho received his B.S. and M.S. degrees from National Taiwan University of Science and Technology in 1988 and 1990 respectively and Ph.D. degree in computer science and information engineering from National Chung Cheng University, Taiwan, ROC in 2003. He is currently a professor in the Department of Applied Digital Media at WuFeng University, Min-Hsiung, Chiayi County, Taiwan. 